\title{
Impactos na saúde humana e no meio ambiente relacionados ao uso de agrotóxicos:
} Uma revisão integrativa

\author{
Impacts on human health and environment related to the use of pesticides: An integrative review \\ Impactos en la salud humana y el medio ambiente relacionados con el uso de plaguicidas: Una \\ revisión integradora
}

Recebido: 24/06/2021 | Revisado: 30/06/2021 | Aceito: 03/07/2021 | Publicado: 15/07/2021

\author{
Cristiana Basso \\ ORCID: https://orcid.org/0000-0003-2001-0110 \\ Universidade Federal de Santa Maria, Brasil \\ E-mail: critianabasso1@gmail.com \\ Anna Carolina Fraga Siqueira \\ ORCID: https://orcid.org/0000-0002-5529-8508 \\ Universidade Franciscana, Brasil \\ E-mail: annacarolinafsq@gmail.com \\ Neila Silvia Pereira dos Santos Richards \\ ORCID: https://orcid.org/0000-0001-6610-5567 \\ Universidade Federal de Santa Maria, Brasil \\ E-mail: neilarichardsprof@gmail.com
}

\begin{abstract}
Resumo
Estudos vêm demonstrando o crescente aumento do uso de agrotóxicos na agricultura convencional no Brasil e no mundo, acarretando em uma maior exposição ao produtor rural, ao consumidor e ao meio ambiente. Com essa preocupação, realizou-se uma revisão integrativa acerca desse tema, nas bases da Web of Science e Scientific Electronic Library (SciELO) e Literatura Latino-Americana e do Caribe em Ciências da Saúde (LILACS), publicadas em português e inglês, entre os anos de 2015 a 2020, que abordaram o tema 'agrotóxicos'. Foram incluídas 35 publicações que demonstraram os impactos na saúde humana e no meio ambiente relacionados ao uso de agrotóxicos. Dos artigos analisados, $89 \%$ foram referentes aos agrotóxicos e efeitos na saúde humana, enquanto apenas $11 \%$ se referiram aos impactos ao meio ambiente. Percebeu-se que a literatura consultada trouxe importantes contribuições, especialmente em se tratando da relação do agrotóxico com a saúde e em contrapartida evidenciou-se pesquisas ainda incipientes voltadas ao meio ambiente.
\end{abstract}

Palavras-chave: Agrotóxicos; Intoxicação; Impactos ambientais e de saúde; Saúde pública.

\begin{abstract}
Studies have shown a growing increase in the use of pesticides in conventional agriculture in Brazil and worldwide, leading to greater exposure of rural producers, consumers and the environment. With this in concern, an integrative review on the topic was carried out in the databases of the Web of Science and Scientific Electronic Library (SciELO) andLatin American and Caribbean Literature on Health Sciences (LILACS), published in Portuguese and English, between the years of 2015 and 2020, which addressed the theme of 'pesticides'. The forums included 35 publications that demonstrate the impacts on human health and on the environment related to the use of pesticides. Of the articles that were analyzed, $89 \%$ were related to pesticides and effects on human health, while only $11 \%$ referred to impacts on the environment. It was noted that there are important contributions of the consulted literature, mainly in dealing with the relationship of agrotoxicity with health and, on the other hand, there is evidence of incipient research aimed at the environment.
\end{abstract}

Keywords: Pesticides; Intoxication; Environmental and health impacts; Public health.

\section{Resumen}

Los estudios han demostrado un aumento creciente en el uso de pesticidas en la agricultura convencional en Brasil y en todo el mundo, lo que lleva a una mayor exposición a los productores rurales, los consumidores y el medio ambiente. Con esta inquietud, se realizó una revisión integradora sobre el tema en las bases de datos de la Web of Science and Scientific Electronic Library (SciELO) y de Literatura Latinoamericana y del Caribe en Ciencias de la Salud (LILACS), publicada en portugués e inglés, entre los años de 2015 a 2020, que abordará el tema de los 'plaguicidas'. Los foros incluyeron 35 publicaciones que demuestran los impactos en la salud humana y el medio ambiente relacionados con el uso de plaguicidas. De los artículos analizados, el $89 \%$ estaban relacionados con plaguicidas y efectos sobre la salud humana, mientras que solo el $11 \%$ se referían a impactos sobre el medio ambiente. Se observó que existen importantes aportes a la literatura consultada, principalmente en el abordaje de la relación de 
plaguicidas con la salud pero, por otro lado, hay evidencias de incipientes investigaciones dirigidas al medio ambiente.

Palabras clave: Plaguicida; Intoxicación; Impacto ambiental y de salud; Salud pública.

\section{Introdução}

Agrotóxicos são produtos químicos sintéticos usados para matar insetos, larvas, fungos, carrapatos, sob a perspectiva de controlar as doenças causadas por esses vetores e de regular o crescimento da vegetação, tanto no ambiente rural quanto urbano. O Brasil vem sendo o país com maior consumo destes produtos desde 2008, decorrente do desenvolvimento do agronegócio no setor econômico (Instituto Nacional de Câncer [INCA], 2019). Esse fato, combinado com os problemas de gerenciamento e controle do consumo por parte dos órgãos ambientais, originou um cenário nacional preocupante, tanto do ponto de vista ambiental, quanto de saúde pública (Souza, da Costa, Maciel, Reis \& Pamplona, 2017).

Frutas e hortaliças consumidas no Brasil por exemplo, contêm elevados níveis de resíduos de defensivos agrícolas que podem representar uma preocupação do ponto de vista de saúde pública (Ferreira, da Silva, Garcia \& Srur, 2018). Assim, preocupada com a saúde coletiva e com o meio ambiente, a Agência Nacional de Vigilância Sanitária (ANVISA) monitora, desde 2001, a qualidade dos alimentos quanto ao uso de agrotóxicos e afins através do Programa de Análise de Resíduos de Agrotóxicos em Alimentos (PARA) (Agência Nacional de Vigilância Sanitária [ANVISA], 2017).

Sabe-se que os impactos adversos da exposição aos agrotóxicos dependem das características químicas, da quantidade absorvida ou ingerida, do tempo de exposição e das condições gerais de saúde da pessoa exposta, sendo divididos em agudos e crônicos (Organização Pan-Americana da Saúde \& Organização Mundial da Saúde [OPAS e OMS], 1996; Rigotto \& Aguiar, 2015). Os efeitos agudos surgem logo após o contato do trabalhador com o agrotóxico e em até 24 horas apresentam sintomas definidos. Os efeitos crônicos são notados em questão de semanas, meses ou anos após o contato com essas substâncias, dificultando a associação desse episódio com o desenvolvimento de patologias, como aquelas que afetam o Sistema Nervoso Central (SNC) e o Sistema Nervoso Periférico (Rigotto \& Aguiar, 2015; Porto \& Soares, 2012).

Desse modo, algumas opiniões são favoráveis ao uso de defensivos agrícolas, pelo fato de que esse grupo de compostos exerce um papel muito importante na contenção de pragas e doenças que podem prejudicar as lavouras, sendo sua aplicação essencial para manter os níveis de produtividade e rentabilidade da atividade agrícola. No entanto, outras opiniões são contrárias, visto que a utilização inadequada de agrotóxicos implica em uma série de ameaças aos agricultores, trabalhadores que os aplicam, habitantes das áreas de produção e consumidores finais dos alimentos colhidos, além de causar danos irreparáveis ao meio ambiente (Días \& Contreras, 2013).

Quanto aos impactos ambientais, estes vão desde a modificação da composição do solo, passando pela contaminação da água e do ar, podendo intervir nos organismos vivos terrestres e aquáticos, adulterando sua morfologia e função dentro do ecossistema (Lopes \& Albuquerque, 2018).

Assim, pesquisas vêm demonstrando o crescente aumento do uso de agrotóxicos na agricultura convencional no Brasil e no mundo, acarretando maior exposição ao produtor rural, ao consumidor e ao meio ambiente. Diante disso, torna-se importante identificar por meio de uma revisão integrativa, quais os impactos que esses insumos químicos têm causado, tanto a saúde humana quanto ao meio ambiente, para dessa forma facilitar a argumentação contra o uso indiscriminado desses insumos.

\section{Metodologia}

O presente estudo trata-se de uma revisão integrativa, com levantamento de dados nas bases da Web of Science e Scientific Electronic Library (SciELO) e Literatura Latino-Americana e do Caribe em Ciências da Saúde (LILACS), das 
produções científicas publicadas em português e inglês a partir de trabalhos realizados entre os anos de 2015 a 2020 que abordaram o tema 'agrotóxicos'.

Adotou-se para nortear a revisão integrativa os trabalhos publicados a respeito dos impactos na saúde humana e no meio ambiente relacionados ao uso de agrotóxicos. A busca de artigos considerou os seguintes termos: 'agrotóxicos', 'defensivos agrícolas', 'resíduo de agrotóxicos', ‘inseticidas', 'agroquímicos', 'insumos químicos', ‘impactos ambientais e de saúde', 'saúde pública', ‘intoxicação', 'segurança alimentar', 'pesticides', 'agriculture', 'farmworkers', 'public health’ e 'occupational exposure'.

Os critérios de inclusão dos estudos foram: (1) que abordassem o tema 'agrotóxicos'; (2) estudos realizados no período de 2015 a 2020; (3) formato de artigo científico; e (4) por último, os estudos que se enquadrassem nos critérios anteriores, mas que abordassem, especificamente os impactos no meio ambiente e na saúde humana relacionados ao uso de agrotóxicos. Foram excluídas as publicações que: (1) não abordaram o tema de interesse do trabalho; (2) estudos publicados anteriormente a 2015; (3) estudos no formato de dissertações, vídeos, livros ou revisões; e (4) estudos repetidos.

\section{Resultados e Discussão}

A busca resultou em 69 artigos e, a partir da análise crítica dos resumos, foram selecionadas 35 publicações que atendiam aos objetivos propostos. Obteve-se um número maior de artigos abrangendo agrotóxicos e a saúde humana, conforme evidencia a Tabela 1.

Após leitura crítica e sistematização dos dados, puderam-se evidenciar dois grupos de discussão: Agrotóxicos e impactos na saúde humana; Impactos ambientais relacionados ao uso de agrotóxicos.

\section{a. Agrotóxicos e impactos na saúde humana}

Para este tópico, foram avaliados 31 artigos (89\%), onde foram discutidos aspectos como: percepção de riscos associados aos agrotóxicos, uso de equipamentos de proteção, relação dos fertilizantes com transtornos mentais, problemas auditivos, doença de Parkinson, malformações congênitas, doenças oncológicas etc. 
Tabela 1. Distribuição dos artigos sobre impactos na saúde humana relacionados aos agrotóxicos publicados de 2015 a 2020.

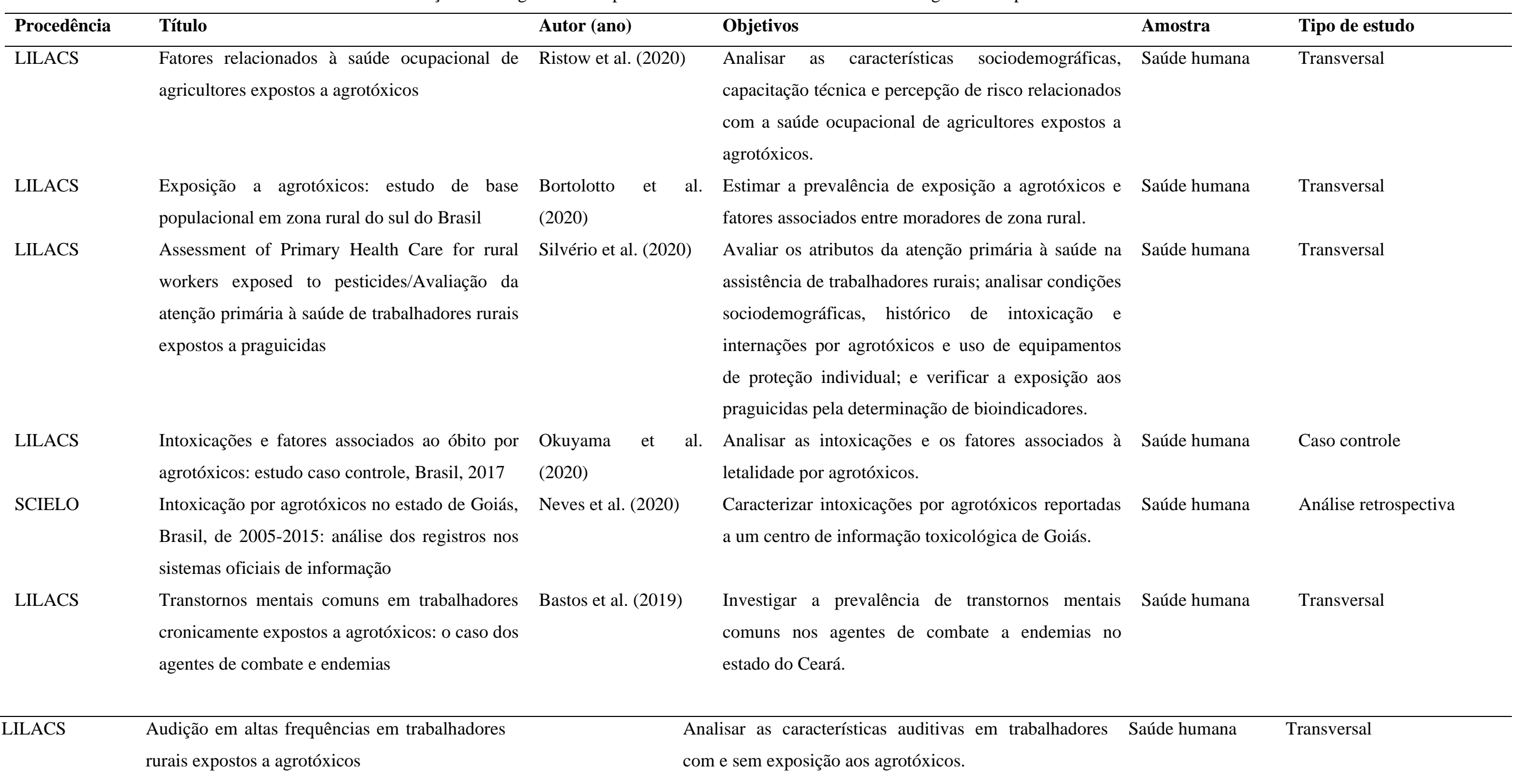


LILACS Epidemiological profile of farmworkers from epidemiológico de trabalhadores rurais do estado do Rio de Janeiro

LILACS

Alterações auditivas de agricultores expostos a agrotóxicos atendidos em um centro especializado em reabilitação

LILACS

LILACS

LILACS Preliminary Study/ A importância da avaliação otoneurológica em trabalhadores brasileiros expostos a pesticidas: um estudo preliminar

Exposição e intoxicação ocupacional a produtos químicos no Distrito Federal the state of Rio de Janeiro/ Perfil

Brust et al. (2019)

Frank

et

The Importance of Otoneurological Evaluation Zeigelboim et al. Verificar os achados da avaliação labiríntica em agentes in Brazilian Workers Exposed to Pesticides: A (2019)

Occupational exposure and poisoning by Magalhães chemical products in the Federal District/ Caldas (2019)

Condições da exposição a agrotóxicos de Vasconcellos portadores da doença de Parkinson al. (2019) acompanhados no ambulatório de neurologia de um hospital universitário e a percepção da relação da exposição com o adoecimento
Descrever o perfil epidemiológico dos trabalhadores rurais expostos a agrotóxicos no município de Casimiro de Abreu, RJ.

al. Determinar a relação entre a exposição à agrotóxicos e as alterações no sistema auditivo de trabalhadores rurais atendidos em um Centro Especializado em Reabilitação Auditiva (CER), Santa Rosa/RS.

Saúde humana

Transversal

de controle de endemias e recomendar a inclusão do exame vestibular no conjunto de exames para populações expostas a agrotóxicos.

e Descrever casos de exposição ocupacional a produtos Saúde humana químicos.

et Investigar, a partir da história laboral de portadores da Saúde humana doença de Parkinson acompanhados no ambulatório de neurologia de um hospital universitário, a ocorrência e as condições da exposição a agrotóxicos.
Saúde humana

Transversal

Saúde humana

Descritivo

Retrospectivo descritivo

Exploratório-descritivo 
residents in the South of Brazil /Exposição

ocupacional a agrotóxicos e alterações

hematológicas: Estudo transversal em

moradores rurais do Sul do Brasil

LILACS

Sistema de Informação de Agravos de

Notificação e as intoxicações humanas por

agrotóxicos no Brasil

LILACS

Câncer infantojuvenil: relação com os polos de irrigação no estado do Ceará

et

acordo com sexo e circunstâncias, no período de 2001 a 2014.

Avaliar a magnitude, tendência espacial e temporal, e Saúde humana fatores de risco relacionados ao câncer infantojuvenil e sua associação com os polos de irrigação no estado do Ceará de 2000 a 2012

Corcino et al. Analisar a influência dos condicionantes sociais, Saúde humana (2019)

a saúde de trabalhadores rurais da fruticultura irrigada

LILACS Análise do impacto do uso de organofosforados (OF) e carbamatos (CAR) em trabalhadores rurais de um município da região noroeste do estado do Rio Grande do Sul

LILACS

LILACS

parâmetros hematológicos em uma população agrícola de

Farroupilha-RS. culturais e econômicos no processo saúde-doença dos trabalhadores expostos a agrotóxicos.

Klein et al. (2018) Analisar o impacto do uso de OF e CAR em Saúde humana trabalhadores rurais na cidade de Mato Queimado/RS.

e Verificar a correlação entre consumo de agrotóxicos em Saúde humana municípios do Paraná e notificação de intoxicações agudas.

\section{Paraná}

Azevedo (2018)

Estimar a prevalência de tremor essencial em um grupo Saúde humana de guardas de endemia expostos cronicamente a

agrotóxicos.

exposta ocupacionalmente a agrotóxicos no

Estado do Rio de Janeiro
Série temporal

Analítico-exploratório

Quantitativo

Transversal

Transversal

Transversal 


\begin{tabular}{|c|c|c|c|c|c|}
\hline LILACS & $\begin{array}{l}\text { Transtornos mentais comuns em agricultores, } \\
\text { relação com agrotóxicos, sintomas físicos e } \\
\text { doenças preexistentes }\end{array}$ & $\begin{array}{l}\text { Morin e Stumm } \\
(2018)\end{array}$ & $\begin{array}{l}\text { Relacionar os transtornos mentais comuns em } \\
\text { agricultores com o uso de agrotóxicos, sintomas físicos, } \\
\text { psíquicos e doenças preexistentes. }\end{array}$ & Saúde humana & Transversal \\
\hline LILACS & $\begin{array}{l}\text { Intoxicações e óbitos por agrotóxicos no Estado } \\
\text { de Goiás, Brasil e inovações legislativas }\end{array}$ & Tejerina $(2018)$ & $\begin{array}{l}\text { Descrever os casos de intoxicações e óbitos por uso de } \\
\text { agrotóxicos no Estado de Goiás e analisar se o Direito à } \\
\text { Saúde está sendo garantido. }\end{array}$ & Saúde humana & Descritivo \\
\hline LILACS & $\begin{array}{l}\text { Intoxicações por agrotóxicos no estado do } \\
\text { Tocantins: 2010-2014 }\end{array}$ & $\begin{array}{l}\text { Silva e Costa } \\
(2018)\end{array}$ & $\begin{array}{l}\text { Caracterizar as intoxicações por agrotóxicos no } \\
\text { Tocantins no período 2010-2014. }\end{array}$ & Saúde humana & Descritivo-exploratório \\
\hline LILACS & $\begin{array}{l}\text { Caracterização epidemiológica de trabalhadores } \\
\text { com câncer em uma região de fruticultura } \\
\text { irrigada }\end{array}$ & $\begin{array}{l}\text { Moura et al. } \\
(2018)\end{array}$ & $\begin{array}{l}\text { Descrever o perfil clínico-epidemiológico dos } \\
\text { trabalhadores com câncer em tratamento em um centro } \\
\text { de oncologia. }\end{array}$ & Saúde humana & $\begin{array}{l}\text { Análise } \\
\text { descritiva }\end{array}$ \\
\hline LILACS & $\begin{array}{l}\text { Saúde do trabalhador e fonoaudiologia: } \\
\text { percepções de agricultores irrigantes expostos a } \\
\text { produtos ototóxicos }\end{array}$ & $\begin{array}{l}\text { Noronha e } \\
\text { Almeida (2017) }\end{array}$ & $\begin{array}{l}\text { Investigar as representações sociais de agricultores rurais } \\
\text { do povoado Várzea dos Cágados na Cidade de Lagarto, } \\
\text { Sergipe, Brasil, sobre as queixas de saúde decorrentes da } \\
\text { exposição a substância ototóxicas e o trabalho do } \\
\text { fonoaudiólogo na saúde desses trabalhadores. }\end{array}$ & Saúde humana & Exploratório \\
\hline LILACS & $\begin{array}{l}\text { Malformações congênitas em regiões de } \\
\text { monocultivo no estado de Minas Gerais, Brasil }\end{array}$ & $\begin{array}{l}\text { Dutra e Ferreira } \\
(2017)\end{array}$ & $\begin{array}{l}\text { Analisar a associação entre o uso de agrotóxicos e as } \\
\text { malformações congênitas em municípios com maior } \\
\text { exposição, e avaliar a natureza da correlação existente } \\
\text { entre a tendência observada e o volume de agrotóxicos } \\
\text { considerados como disruptores endócrinos. }\end{array}$ & Saúde humana & Transversal \\
\hline LILACS & $\begin{array}{l}\text { Implicações do uso de agrotóxicos: percepções } \\
\text { de familiares de crianças portadores de } \\
\text { neoplasia }\end{array}$ & $\begin{array}{l}\text { Camponogara et } \\
\text { al. (2017) }\end{array}$ & $\begin{array}{l}\text { Conhecer as percepções de familiares de crianças } \\
\text { portadoras de neoplasia, atuantes como trabalhadores } \\
\text { rurais, acerca do processo de adoecimento e implicações }\end{array}$ & Saúde humana & Análise temática \\
\hline
\end{tabular}


do uso de agrotóxicos.

\begin{tabular}{|c|c|c|c|c|c|}
\hline SCIELO & $\begin{array}{l}\text { Distribuição espacial do uso de agrotóxicos no } \\
\text { Brasil: uma ferramenta para a Vigilância em } \\
\text { Saúde }\end{array}$ & $\begin{array}{l}\text { Pignati et al. } \\
(2017)\end{array}$ & $\begin{array}{l}\text { Apresentar a distribuição espacial da área plantada de } \\
\text { lavouras, consumo de agrotóxicos e agravos à saúde } \\
\text { relacionados, como estratégia de Vigilância em Saúde. }\end{array}$ & Saúde humana & Epidemiológico \\
\hline SCIELO & $\begin{array}{l}\text { Conhecimentos, atitudes e práticas de } \\
\text { agricultoras sobre o processo de produção de } \\
\text { tabaco em um município da Região Sul do } \\
\text { Brasil }\end{array}$ & Reis et al. (2017) & $\begin{array}{l}\text { Compreender os conhecimentos, atitudes e práticas de } \\
\text { agricultoras que trabalham no processo de produção do } \\
\text { tabaco sobre os impactos sociais, ambientais e à saúde, } \\
\text { decorrentes desta atividade econômica. }\end{array}$ & Saúde humana & Exploratório-qualitativo \\
\hline LILACS & $\begin{array}{l}\text { Sociedade de risco: o uso dos agrotóxicos e } \\
\text { implicações na saúde do trabalhador rural }\end{array}$ & Viero et al. (2016) & $\begin{array}{l}\text { Conhecer as percepções de trabalhadores rurais sobre os } \\
\text { riscos advindos do uso de agrotóxicos para sua saúde. }\end{array}$ & Saúde humana & Descritivo \\
\hline LILACS & $\begin{array}{l}\text { Saúde e ambiente nas políticas públicas em } \\
\text { municípios que cultivam tabaco no sul do } \\
\text { Brasil }\end{array}$ & $\begin{array}{l}\text { Santos et al. } \\
(2015)\end{array}$ & $\begin{array}{l}\text { Analisar os desafios e as potencialidades para o } \\
\text { desenvolvimento e a implementação de Políticas } \\
\text { Públicas Locais que enfoquem a relação entre o uso do } \\
\text { agrotóxico, a saúde da população rural e do ambiente em } \\
\text { municípios produtores de tabaco do sul do Brasil. } \\
\text { Explorar correlações entre variáveis relativas à produção }\end{array}$ & Saúde humana & Qualitativo \\
\hline SCIELO & $\begin{array}{l}\text { Correlação entre produção agrícola, variáveis } \\
\text { clínicas-demográficas e câncer de próstata: um } \\
\text { estudo ecológico }\end{array}$ & Silva et al. (2015) & $\begin{array}{l}\text { agrícola, ao uso de serviços de saúde e } \\
\text { sociodemográficas e às taxas de mortalidade por câncer } \\
\text { de próstata entre 2005-2009, nos estados brasileiros. }\end{array}$ & Saúde humana & Exploratório \\
\hline
\end{tabular}


Fica evidente a utilização rotineira de agrotóxicos no meio agrícola e que a exposição a estes agentes químicos pode afetar a saúde do trabalhador, principalmente pela mistura de vários grupos químicos, que podem interagir entre si e acarretar impactos adversos à saúde (Sena, Dourado \& Antoniolli, 2019). O uso indiscriminado de agrotóxicos tem provocado um número significativo de intoxicações, tanto agudas quanto crônicas (Frank, Caye, Mattiazzi \& Battisti, 2019).

$\mathrm{Na}$ intoxicação aguda, os sintomas surgem imediatamente após a exposição ao agrotóxico (Noronha \& Almeida, 2017). Os sintomas mais frequentes causados por este tipo de intoxicação são identificados por náuseas, vômitos, cefaleia, fadiga, visão embaçada, desorientação, dores no peito, taquicardia, dificuldade respiratória, vertigem, irritação na pele, olhos e mucosas (Noronha \& Almeida, 2017; Taveira \& Albuquerque, 2018). Em relação à intoxicação crônica, o surgimento de efeitos no indivíduo acontece de forma tardia e pode aparecer após meses ou anos da exposição aos agentes tóxicos (Noronha \& Almeida, 2017; Azevedo et al., 2018).

Como os sintomas da intoxicação aguda são muito inespecíficos, muitas vezes os agricultores não os correlacionam ao uso de agrotóxicos, contribuindo assim para a subnotificação dos casos, como mostrou um estudo em que apesar de $30 \%$ dos trabalhadores rurais entrevistados terem apresentado sintomas de intoxicação aguda em algum momento durante a aplicação de agrotóxicos, apenas $7 \%$ foram diagnosticados com intoxicação aguda por agrotóxicos, provavelmente devido à baixa procura e à falha no diagnóstico (Taveira \& Albuquerque, 2018).

Assim a formulação de indicadores de saúde com dados de intoxicação por agrotóxicos torna-se um desafio, acerca da elevada subnotificação destes agravos. Para cada caso registrado de intoxicação aguda, outros 50 não são notificados (Pignati, et al.,2017).

Noronha e Almeida (2017) constataram que os fatores de risco dos trabalhadores em relação à intoxicação por agrotóxicos decorrem, em alguns casos, do modo de produção agrícola, das condições de trabalho, da falta de orientação e inadequações no manejo dos produtos. O baixo nível de escolaridade dos indivíduos envolvidos na agricultura foi observado em diversos trabalhos (Ristow, Battisti, Stumm \& Montagner, 2020; Moura, Aninger, Barbosa \& Bedor, 2018).

Em estudo realizado por Corcino et al. (2019), o maior índice de intoxicações ocorreu entre os não alfabetizados, evidenciando que socialmente esta condição deve ser levada em consideração. Em contrapartida, Vasconcellos, Rizzotto, Machineski e Costa (2019) verificaram que a baixa escolaridade pode dificultar a leitura e o entendimento sobre os efeitos nocivos dos agrotóxicos, no entanto, não pode ser considerada como fator isolado para o seu uso incorreto.

Outra constatação alcançada com os dados de notificações por agrotóxicos foi de que o maior número de intoxicações ocorre em homens (Neves, Mendonça, Bellini \& Pôssas, 2020). Silvério et al. (2020) verificaram que mulheres acabam tendo uma exposição a concentrações mais baixas, o que explica a menor ocorrência de intoxicações nesse gênero. Vários estudos corroboram com o fato que o sexo masculino apresenta o maior percentual dos casos notificados, possivelmente devido aos homens desenvolverem atividades que possuem um contato mais direto e em concentrações maiores a agrotóxicos, estando assim mais expostos a esses produtos, em relação às mulheres (Noronha \& Almeida, 2017; Ristow, Battisti, Stumm, Montagner, 2020; Silvério et al., 2020; Magalhães \& Caldas, 2019; Corcino et al., 2019; Neves, Mendonça, Bellini \& Pôssas, 2020; Silva \& Costa, 2018).

Essa exposição só aumenta, visto que nos últimos anos o Brasil se tornou o maior consumidor mundial de agrotóxicos (Tejerina, 2018). Neste enredo com expressivos comprometimentos à saúde do agricultor, ressaltam-se os danos à saúde mental desta população (Morin, Stumm, 2018) devido a intoxicação crônica por anos de exposição a agrotóxicos, tanto de forma ocupacional, ambiental ou acidental (ingestão) (Neves, Mendonça, Bellini \& Pôssas, 2020). Esses agentes estão envolvidos entre 10 e $20 \%$ dos suicídios no mundo (Okuyama, Galvão \& Silva, 2020).

Neves, Mendonça, Bellini, Pôssas (2020) e outros pesquisadores (Silva, Costa, 2018; Tejerina, 2018) apontam a 
tentativa de suicídio como sendo a intoxicação por agrotóxico com maior ocorrência. Este resultado está atrelado a uma intoxicação crônica de alta exposição na aplicação de agrotóxicos que também pode causar problemas imunológicos, hematológicos, hepáticos, neurológicos, malformações congênitas, cânceres (Neves, Mendonça, Bellini \& Pôssas, 2020; Silva \& Costa, 2018; Tejerina, 2018) e a ocorrência de alterações especificamente nos sistemas e vias auditivas e vestibular (Noronha \& Almeida, 2017).

Outros autores também referem que o uso constante de agrotóxicos na agricultura tem provocado uma série de complicações em relação à saúde, como, por exemplo, distúrbios auditivos (Noronha \& Almeida, 2017; Zeigelboim et al., 2019). Estes que afetam a função auditiva são chamados de agentes ototóxicos, ou seja, são substâncias que causam danos funcionais ou lesões celulares na orelha interna, especialmente nas frequências agudas (3000 Hz e $6000 \mathrm{~Hz})$ (Noronha \& Almeida, 2017).

Entre os diversos impactos à saúde gerados pelo uso intensivo de agrotóxicos, pode-se destacar a ocorrência de doenças e mortes que poderiam ser evitadas, entre elas o câncer (Camponogara et al., 2017). A exposição a estas substâncias químicas em regiões de média e alta produção agrícola tem sido apontada como potenciais fatores causais de cânceres, uma vez que a Agência Internacional de Pesquisa em Câncer (IARC/OMS) tem classificado agrotóxicos frequentemente utilizados nas lavouras como potencialmente cancerígenos, por exemplo, o glifosato (Pignati et al., 2017).

Neste contexto, destaca-se o surgimento de câncer em agricultores, com origem nos sistemas digestório, reprodutor masculino, imunológico, endócrino, tegumentar, respiratório e urinário (Morin \& Stumm, 2018). Silva, Silva, Lima-Luz, Aydos e Mattos (2015) observam associação entre exposição a agrotóxicos e câncer de próstata nas últimas duas décadas. Em conformidade, Moura, Aninger, Barbosa e Bedor (2018) demonstram um aumento de mortalidade por doenças neoplásicas em trabalhadores rurais, sendo que, as principais taxas de mortalidade por câncer estavam relacionadas às neoplasias malignas de próstata e pulmão. Corrobora com esses dados a situação atual do Brasil, em que o câncer vem ganhando relevância pelo perfil epidemiológico, implicando em desafio aos profissionais e serviços de saúde (Camponogara et al., 2017).

A comunidade científica tem abordado as inúmeras modificações provocadas pela intoxicação por agrotóxicos no ser humano, responsáveis por desencadear uma série de problemas à saúde (Noronha \& Almeida, 2017). Além dos impactos já abordados, Piccoli, Cremonese, Koifman, Koifman, e Freire (2019) associam a exposição humana a agrotóxicos a vários efeitos nocivos à saúde, incluindo distúrbios endócrinos, defeitos de nascença, efeitos neurológicos, hepáticos, respiratórios e imunológicos.

Bortolotto, Hirschmann, Martins-Silva e Facchini (2020) ainda mencionam que os agrotóxicos, quando empregados de maneira indiscriminada, podem causar desde sintomas mais leves, como dermatites, até doenças crônicas não transmissíveis, parto prematuro, infertilidade masculina, doença de Parkinson e Alzheimer.

Em concordância, Neves, Mendonça, Bellini e Pôssas (2020) reforçam que alguns agrotóxicos causarão 50\% do autismo em crianças até 2025, e outras doenças modernas, como depressão, infertilidade, Alzheimer, câncer e doenças cardíacas. Como a produção agrícola brasileira é dependente da utilização de fertilizantes químicos (Silva, Silva, Lima-Luz, Aydos \& Mattos; 2015), cabe discutir cada vez mais as formas de exposição aos agrotóxicos e seus impactos na saúde humana.

\section{b. Impactos ambientais relacionados ao uso de agrotóxicos}

Para este tópico, foram avaliados 4 artigos (11\%), onde foram discutidos aspectos referentes aos agrotóxicos e seus impactos na natureza. Embora o meio ambiente tenha relação direta com a saúde humana, de forma específica a relação entre uso de agrotóxicos e seus impactos ao meio ambiente ainda é incipiente, conforme evidencia a Tabela 2. 
Tabela 2. Distribuição dos artigos sobre impactos no meio ambiente relacionados aos agrotóxicos publicados de 2015 a 2020.

\begin{tabular}{|c|c|c|c|c|c|}
\hline LILACS & $\begin{array}{l}\text { A extensão do "agro" e do tóxico: saúde e } \\
\text { ambiente na terra indígena Marãiwatsédé, Mato } \\
\text { Grosso }\end{array}$ & $\begin{array}{l}\text { Souza e Lima et } \\
\text { al. (2020) }\end{array}$ & $\begin{array}{l}\text { Verificar resíduos de agrotóxicos na água e discutir a } \\
\text { dinâmica de inserção da agropecuária na TI e região. }\end{array}$ & Meio ambiente & Quantitativo \\
\hline LILACS & $\begin{array}{l}\text { Estimativa de contaminação de águas } \\
\text { subterrâneas e superficiais por agrotóxicos em } \\
\text { área sucroalcooleira, Santa Rita/PB, Brasil }\end{array}$ & $\begin{array}{l}\text { Ismael e Rocha } \\
\text { (2019) }\end{array}$ & $\begin{array}{l}\text { Estimar o potencial de contaminação das águas } \\
\text { subterrâneas e superficiais por agrotóxicos aplicados em } \\
\text { uma área sucroalcooleira na região metropolitana de } \\
\text { João Pessoa, município de Santa Rita/PB. }\end{array}$ & Meio ambiente & Descritivo \\
\hline LILACS & $\begin{array}{l}\text { Processo sócio-sanitário-ambiental da poluição } \\
\text { por agrotóxicos na bacia dos rios Juruena, } \\
\text { Tapajós e Amazonas em Mato Grosso, Brasil }\end{array}$ & $\begin{array}{l}\text { Oliveira et al. } \\
\text { (2018) }\end{array}$ & $\begin{array}{l}\text { Compreender o processo de poluição ambiental por } \\
\text { agrotóxicos nos municípios de Campo Novo do Parecis, } \\
\text { Sapezal e Campos de Júlio, em Mato Grosso, Brasil. }\end{array}$ & Meio ambiente & Interpretativo integrado \\
\hline SCIELO & $\begin{array}{l}\text { Agricultura familiar e a conservação da saúde } \\
\text { humana e ambiental }\end{array}$ & $\begin{array}{l}\text { Borges et al. } \\
(2016)\end{array}$ & $\begin{array}{l}\text { Compreender como os trabalhadores agricultores } \\
\text { identificam os impactos positivos e negativos do trabalho } \\
\text { agrícola familiar no ambiente e conservam a saúde } \\
\text { ambiental. }\end{array}$ & Meio ambiente & Qualitativo \\
\hline
\end{tabular}


Com a inserção de novas tecnologias na produção agrícola, os estabelecimentos agropecuários tiveram a necessidade de inserir substâncias químicas nas atividades agrícolas. Vale ressaltar que a mudança no processo produtivo contribuiu para o desequilíbrio ambiental, provocando impactos e degradação da biosfera (Noronha \& Almeida, 2017). No meio ambiente os agrotóxicos causam a contaminação do solo, poluição dos rios, além de persistirem nas cadeias tróficas (Dutra \& Ferreira, 2017; Viero, Camponogara, Cezar-Vaz, Costa \& Beck, 2016).

O debate em torno do uso de agrotóxicos na produção agrícola é discutido por diferentes campos do conhecimento, desde aqueles relacionados ao desenvolvimento econômico e social, até o campo da saúde. Nesse último, considera-se que há uma contaminação crescente de solos e águas, uma diminuição da biodiversidade e o adoecimento do trabalhador rural (Santos, Ruiz, Riquinho \& Mesquita, 2015). No entanto, não são somente os agricultores em suas atividades que estão expostos aos agrotóxicos, mas sim toda população devido a fatores como contaminação dos recursos naturais e dos alimentos (Camponogara et al., 2017; Silva, Silva, Lima-Luz \& Mattos, 2015).

O modelo produtivo monocultor do agronegócio requer a intensa utilização de agrotóxicos, a qual compromete o ambiente, estando o mesmo interrelacionado com a saúde humana (Barbosa, Sales, Arregi \& Rigotto, 2019). O reconhecimento dos impactos ao meio ambiente para a saúde tem sido percebido com maior visibilidade junto às agendas de atuação no campo da saúde. Ainda assim, há negligência na atuação sobre os determinantes ambientais na saúde, principalmente no que tange a redução dos riscos ambientais, incluindo a exposição ao ar e águas contaminadas por resíduos químicos ou devido à insuficiência no acesso ao saneamento básico (Santos, Ruiz, Riquinho \& Mesquita, 2015).

A presença de agrotóxicos no ambiente é uma preocupação para a saúde pública e seu monitoramento tem se tornado frequente (Souza e Lima, Pignati, \& Pignatti, 2020). Em contrapartida, na Portaria do Ministério da Saúde n $2914 / 2011$ encontra-se regulamentado 64 substâncias químicas que representam riscos à saúde humana, dentre as quais 27 são agrotóxicos, o que de acordo com a Associação Brasileira de Saúde Coletiva (ABRASCO), este número representa menos de 10\% dos princípios ativos oficialmente registrados no país (Ismael \& Rocha, 2019).

O impacto direto da contaminação humana por agrotóxicos é percebido por meio de três vias principais: ocupacional, ambiental e alimentar (Souza e Lima, Pignati, \& Pignatti, 2020; Ismael \& Rocha, 2019). A via ambiental é caracterizada pela dispersão e dinâmica dos resíduos de agrotóxicos aos diferentes compartimentos ambientais. Essas poluições podem ocorrer através de correntes aéreas por meio de pulverizações e infiltração no solo chegando até os lençóis freáticos, já sendo detectados no ar, na chuva, na água e em animais (Vascondellos, Rizzotto, Machineski \& Costa, 2019).

As percepções acima remetem aos problemas ambientais decorrentes do uso de agrotóxicos (Camponogara et al., 2017). Inúmeras substâncias têm sido processadas e lançadas ao meio ambiente pela indústria química para alavancar a produção agrícola e a indústria de transformação (Souza e Lima, Pignati \& Pignatti, 2020). Segundo Ismael e Rocha (2019), alguns tipos de agrotóxicos, ao permanecerem no ambiente ou alcançarem o meio aquático, oferecem riscos para espécies animais por sua toxicidade e possibilidade de bioacumulação na cadeia alimentar.

Na cadeia produtiva do agronegócio, as poluições, os agravos e as doenças relacionadas aos agrotóxicos têm se apresentado como um dos impactos de maior relevância para a saúde do trabalhador, da população e do ambiente. A poluição ambiental por agrotóxicos, portanto, está inserida como componente da determinação do perfil epidemiológico ou do processo saúde-doença da população em regiões produtivas do agronegócio (Oliveira, Pignati, Pignatti, Beserra \& Leão, 2018).

Embora existam padrões de segurança para o uso de agrotóxicos, a forma como isto é feito tem causado contaminações ambientais e provocado agravos à saúde da população exposta (Souza e Lima, Pignati \& Pignatti, 2020). Para Borges, Bonow, Silva, Rocha e Cezar-Vaz, (2016) o manejo intensivo dos agrotóxicos, mesmo em total observância às leis/normas, leva à poluição dos alimentos, das águas, do ar, das chuvas, do trabalhador, da população e dos animais, ou seja, contamina as formas 
de vida vinculadas a essas substâncias.

Diante dessas informações, a identificação e avaliação de riscos à saúde e ao meio ambiente se tornam importantes ferramentas para contribuir com o controle e prevenção da exposição da população aos agrotóxicos (Ismael \& Rocha, 2019).

\section{Conclusão}

Mediante análise dos artigos selecionados, percebeu-se que a literatura trouxe importantes contribuições, especialmente em se tratando da relação do agrotóxico com a saúde humana, em contrapartida evidenciou-se pesquisas ainda incipientes voltadas especificamente ao meio ambiente.

Outro aspecto que merece maior pesquisa é referente a formas de diminuir o agrotóxico presente no alimento, além da higienização adequada.

\section{Referências}

Agência Nacional de Vigilância Sanitária. (2017). Programa de análise de resíduos de agrotóxicos em alimentos (Para): relatório de atividades de 2011 e 2012. Anvisa. https://www.gov.br/anvisa/pt-br/assuntos/agrotoxicos/programa-de-analise-de-residuos-em-alimentos.

Azevedo, M. F. A de. (2018). Prevalência do tremor essencial em população exposta ocupacionalmente a agrotóxicos no estado do Rio de Janeiro. Revista Brasileira de Neurologia, 54(1), 10-15.

Barbosa, I. M., Sales, D. S., Arregi, M., U., \& Rigotto, R. M. (2019). Câncer infantojuvenil: relação com os polos de irrigação agrícola no estado do Ceará, Brasil. Ciência \& Saúde Coletiva, 24(4), 1563-1570.

Bastos, M. L. A., Carvalho, M. C. L de., Ferreira, M. J. M., Freitas, T. H., \& Silva-Junior, G. B da. (2019). Transtornos mentais comuns em trabalhadores cronicamente expostos a agrotóxicos: o caso dos agentes de combate a endemias. Revista Brasileira de Medicina do Trabalho, 17(4), 506-510.

Borges, A. M., Bonow, C. A., Silva, M. R. S da., Rocha, L. P., Cezar-Vaz, M.R. (2016). Agricultura familiar e a conservação da saúde humana e ambiental. Revista Brasileira de. Enfermagem, 69(2), 326-334.

Bortolotto, C. C., Hirschmann, R., Martins-Silva T., \& Facchini, L. A. (2020). Exposição a agrotóxicos: estudo de base populacional em zona rural do sul do Brasil. Revista Brasileira de Epidemiologia, 23, 1-11.

Brust, R. S, Oliveira, L. P. M de., Silva, A. C. S. S da., Regazzi, I, C. R., Aguiar, G. S de., Knupp, V. M de, A. O. (2019). Epidemiological profile of farmworkers from the state of Rio de Janeiro. Revista Brasileira de Enfermagem, 72,122-128.

Camponogara, S., Correio, I. L de. L. R., Dias, G. L., Correio, L. N. M., Correio, C. M. V. \& Correio, J. D. M. (2017). Implicações do uso de agrotóxicos: Percepções de familiares de crianças portadoras de neoplasia. Revista Online de Pesquisa: Cuidado é Fundamental, 9(3), 786-794.

Corcino, C. O., Teles, R. B. de A., Almeida, J. R. G. da S., Lirani, L. da S., Araújo, C. R. M., Gonsalves., A. de A., \& Maia, G. L. de A. (2019). Avaliação do efeito do uso de agrotóxicos sobre a saúde de trabalhadores rurais da fruticultura irrigada. Ciência \& Saúde Coletiva, 24(8), 3117-3128.

Días, P. B., Contreras L. M. (2013). Poluição de águas superficiais por resíduos de pesticidas na Venezuela ou em outros países da América Latina. Revista internacional de contaminação ambiental, 29, 7-23.

Dutra, L. S., Ferreira, A. P. (2017). Malformações congênitas em regiões de monocultivo no estado de Minas Gerais, Brasil. Medicina (Ribeirão Preto), 50(5), 285-296.

Ferreira, V. B, da Silva, T. T. C., Garcia, S. R. M. C., \& Srur, A. U. O. S. (2018). Estimativa de ingestão de agrotóxicos organofosforados pelo consumo de frutas e hortaliças - Caderno de Saúde Coletiva, 26 (2), 216-221.

Frank, J. G., Caye, J. L., Mattiazzi, A. L., Battisti, I. D. E. (2019). Alterações auditivas de agricultores expostos a agrotóxicos atendidos em um centro especializado em reabilitação. Revista Brasileira de Ciências da Saúde, 23(4), 471-484.

Instituto Nacional de Câncer. (2019). Ministério da Saúde. Agrotóxicos. INCA.

Ismael, L. L., \& Rocha, E. M. R. (2019). Estimativa de contaminação de águas subterrâneas e superficiais por agrotóxicos em área sucroalcooleira, Santa Rita/PB, Brasil. Ciência \& Saúde Coletiva, 24(12), 4665-4675.

Klein, B. N., Staudt, K. J., Missio, R., Peruzzi, H. M., \& Almeida, I. A. (2018). Análise do impacto do uso de organofosforados e carbamatos em trabalhadores rurais de um município da região noroeste do estado do Rio Grande do Sul. Acta Toxicológica Argentina, 26(3).

Lopes, C. V. A., Albuquerque, G. S. C. de. (2018). Agrotóxicos e seus impactos na saúde humana e ambiental: uma revisão sistemática. Saúde Debate, 42(117), 518-534.

Magalhães, A. F. A, Caldas, E. D. (2019). Exposição e intoxicação ocupacional a produtos químicos no Distrito Federal. Revista Brasileira de. Enfermagem, 72(suppl 1), 32-40. 
Morin, P. V., Stumm, E. M. F. (2018). Transtornos mentais comuns em agricultores, relação com agrotóxicos, sintomas físicos e doenças preexistentes. Psicologia, 49(2), 196-205.

Moura, L. T. R de., Aninger, P. R. L. de C., Barbosa, A. V., Bedor, C. N. G. (2018). Caracterização epidemiológica de trabalhadores com câncer em uma região de fruticultura irrigada. Revista Baiana de Saúde Pública, 42(1), 7-25.

Neves, P. D. M., Mendonça, M. R., Bellini, M., Pôssas, I. B. (2020). Intoxicação por agrotóxicos agrícolas no estado de Goiás, Brasil, de 2005-2015: análise dos registros nos sistemas oficiais de informação. Ciência \&.Saúde Coletiva, 25(7), 2743-2754.

Noronha, M. S. de M., Almeida, M. E de. (2017). Saúde do trabalhador e fonoaudiologia: percepções de agricultores irrigantes expostos a produtos ototóxicos. Revista Baiana de Saúde Pública, 41(4), 947-964.

Okuyama, J. H. H., Galvão, T. F., Silva, M. T. (2020). Intoxicações e fatores associados ao óbito por agrotóxicos: estudo caso controle, Brasil, 2017. Revista Brasileira de Epidemiologia, 23, 1-13.

Oliveira, L. K de., Pignati, W., Pignatti, M. G., Beserra, L., \& Leão, L. H da. C. (2018). Processo sócio-sanitário-ambiental da poluição por agrotóxicos na bacia dos rios Juruena, Tapajós e Amazonas em Mato Grosso, Brasil. Saúde e Sociedade, 27(2), 573-587.

Organização Pan-Americana da Saúde. Organização Mundial da Saúde. (1996). Manual de Vigilância da Saúde de Populações Expostas a Agrotóxicos. Brasília: Opas/OMS. https://bvsms.saude.gov.br/bvs/publicacoes/livro2.pdf.

Piccoli, C., Cremonese, C., Koifman, R., Koifman, S., \& Freire, C. (2019). Exposição ocupacional a agrotóxicos e alterações hematológicas: estudo transversal em moradores rurais do Sul do Brasil. Ciência \& Saúde Coletiva, 24(6), 2325-2340.

Pignati, W. A., Lima, F. A. N. de S., Lara, S. S. de., Correa, M. L. M., Barbosa, J. R., Leão, L. H. da C. (2017). Distribuição espacial do uso de agrotóxicos no Brasil: uma ferramenta para a Vigilância em Saúde. Ciência \& Saúde Coletiva, 22(10), 3281-3293.

Porto, M. F., Soares, W. L. (2012). Modelo de desenvolvimento, agrotóxicos e saúde: um panorama da realidade agrícola brasileira e propostas para uma agenda de pesquisa inovadora. Revista brasileira de saúde ocupacional, 37(125), 17-31.

Queiroz, P. R., Lima, K. C., Oliveira, T. C de., Santos, M. M dos., Jacob, J. F., \& Oliveira, A. M. B.M. de. (2019). Sistema de Informação de Agravos de Notificação e as intoxicações humanas por agrotóxicos no Brasil. Revista Brasileira de Epidemiologia, 22, 1-10.

Reis, M. M dos., Oliveira, A. P. N de., Turci, S. R. B., Dantas, R. M., Silva, V dos. S. S. P da., Gross, C., Jensen, T., \& Silva, V. L da C. e. (2017). Conhecimentos, atitudes e práticas de agricultoras sobre o processo de produção de tabaco em um município da Região Sul do Brasil. Cadernos de saúde pública, 33(sup3), 148-161.

Rigotto, R. M., Aguiar, A. C. P. (2015). Invisibilidade ou invisibilização dos efeitos crônicos dos agrotóxicos à saúde? Desafios à ciência e às políticas públicas in Observatório Internacional de Capacidades Humanas, Desenvolvimento e Políticas Públicas: estudos e análises 2. Brasília, 47-90. http://capacidadeshumanas.org/oichsite/wp-content/uploads/2015/06/03_agrotoxicos-final.pdf

Ristow, L. P., Battisti, I. D. E., Stumm, E. M. F., Montagner, S. E. D. (2020). Fatores relacionados à saúde ocupacional de agricultores expostos a agrotóxicos. Saúde e sociedade, 29(2), 1-11.

Santos, V. C. F dos., Ruiz, E. N. F., Riquinho, D. L., Mesquita, M. O. (2015). Saúde e ambiente nas políticas em municípios que cultivam tabaco no sul do Brasil. Revista Gaúcha de Enfermagem, 36, 215-223.

Silva, J. F. S. da., Silva, A. M. C da., Lima-Luz, L., Aydos, R. D. \& Mattos, I. E. (2015). Correlação entre produção agrícola, variáveis clínicas-demográficas e câncer de próstata: um estudo ecológico. Ciência \& Saúde Coletiva, 20(9), 2805- 2812.

Silva, S. L de O., Costa E. A. (2018). Intoxicações por agrotóxicos no estado do Tocantins: 2010-2014. Visa em Debate, 6(4), 13-22.

Silvério, A. C. P., Martins, I., Nogueira, D. A., Mello, M. A. S., Loyola, E. A. C de., \& Graciano, M. M. de C. (2020). Avaliação da atenção primária à saúde de trabalhadores rurais expostos a praguicidas. Revista de Saúde Pública, 54(9), 1-11.

Souza e Lima, F. A. N de., Pignati, W. A., \& Pignatti, M. G. (2020). A extensão do 'agro' e do tóxico: saúde e ambiente na terra indígena Marãiwatsédé, Mato Grosso. Cadernos Saúde Coletiva, 28(1), 1-11.

Souza, G. S., da Costa, L. C. A., Maciel, A. C., Reis, F. D. V., \& Pamplona, Y. A. P. (2017). Presença de agrotóxicos na atmosfera e risco à saúde humana: uma discussão para a Vigilância em Saúde Ambiental - Ciência \& Saúde Coletiva, 22 (10), 3269-3280.

Taveira, B. L. S., Albuquerque, G. S. C de. (2018). Análise das notificações de intoxicações agudas, por agrotóxicos, em 38 municípios do estado do Paraná. Saúde em debate, 42(4), 211-222.

Tejerina G. R. de L. (2018). Intoxicações e óbitos por agrotóxicos no Estado de Goiás, Brasil e inovações legislativas. Cadernos Ibero-Americanos de Direito Sanitário, 7(1), 229- 249 .

Vasconcellos, P. R. O., Rizzotto, M. L. F., Machineski, G. G., Costa, R. M. (2019). Condições da exposição a agrotóxicos de portadores da doença de Parkinson acompanhados no ambulatório de neurologia de um hospital universitário e a percepção da relação da exposição com o adoecimento. Saúde em debate, 43(123), 1084-1094.

Viero, C. M., Camponogara, S., Cezar-Vaz, M. R., Costa, V. Z da., Beck, C. L. C. (2016). Sociedade de risco: o uso dos agrotóxicos e implicações na saúde do trabalhador rural. Escola Anna Nery, 20(1), 99-105.

Zeigelboim, B. S., Malisky, J. S., Rosa, M. R da., Lacerda, A. B. M de., Alcaraz, P. de S. \& Fonseca, V. R. (2019). A importância da avaliação otoneurológica em trabalhadores brasileiros expostos a agrotóxicos: Um estudo preliminar. International Archives of Otorhinolaryngology, 23(4), 389-395. 